\title{
DECOMPOSING CUBES
}

\section{P. HORAK, J. ŠIRÁŇ and W. WALLIS}

(Received 8 December 1994)

Communicated by L. Caccetta

\begin{abstract}
A graph $H$ decomposes into a graph $G$ if one can write $H$ as an edge-disjoint union of graphs isomorphic to $G$. $H$ decomposes into $D$, where $D$ is a family of graphs, when $H$ can be written as a union of graphs each isomorphic to some member of $D$, and every member of $D$ is represented at least once. In this paper it is shown that the $d$-dimensional cube $Q_{d}$ decomposes into any graph $G$ of size $d$ each of whose blocks is either an even cycle or an edge. Furthermore, $Q_{d}$ decomposes into $D$, where $D$ is any set of six trees of size $d$.
\end{abstract}

1991 Mathematics subject classification (Amer. Math. Soc.): primary 05C70; secondary 05C 99.

\section{Introduction}

We use the standard ideas of graph theory. All graphs are finite, simple and undirected. A graph $H$ decomposes into a graph $G$ if $H$ can be written as an edge-disjoint union of copies of $G$.

It has been thought for a long time that the general graph decomposition problem is hard. This was confirmed when Dolinski and Tarsi [1] proved that unless $G$ is of the form $t K_{2} \cup n P_{3}$, the $G$-decomposition problem is NP-complete. In view of their result it is not surprising that there is an interest in restricted decomposition problems. One of the most famous conjectures is that of Ringel [4]:

CONJECTURE. The complete graph on $2 n+1$ vertices decomposes into any tree of size $n$.

Many partial results have been obtained and recently an analogue of the conjecture has been proved independently by Fink [2] and Jacobson, Truszczynski and Tuza [3].

(C) 1996 Australian Mathematical Society 0263-6115/96 \$A2.00+0.00 
THEOREM. ([2,3]) The d-dimensional cube $Q_{d}$ decomposes into any tree of size $d$.

The Theorem can be generalized in three ways: one can replace 'cube' by a more general graph, replace 'tree' by a more general graph, or consider decompositions into families rather than decompositions into a single graph. In [3] the following generalization of the first kind is proposed.

CONJECTURE. Every $d$-regular bipartite graph decomposes into any tree of size $d$.

In the present paper we focus on a generalization of the second kind, namely we show that if the graph $G$ of size $d$ has the property that any block is either an even cycle or an edge then $Q_{d}$ decomposes into $G$. In view of this result and other supporting evidence we believe that the following conjecture could be true.

CONJECTURE. If $G$ is a graph of size $d$ embeddable into $Q_{d}$, then $Q_{d}$ decomposes into $G$.

This conjecture is of course an analog of Wilson's Theorem [5] that for fixed $\lambda$ and $G$, the $\lambda$-fold complete multigraph $K_{n}^{(\lambda)}$ decomposes into $G$ provided $n$ is sufficiently large and the obvious divisibility conditions hold.

In [2] Fink discusses a generalization of the third kind. Let $F$ be a set of graphs. Then it is said that there is an $F$-decomposition of a graph $G$ if $G$ can be partitioned into subgraphs each of which is isomorphic to a member of $F$ such that every graph from $F$ is represented at least once in the decomposition of $G$. He asks what is the largest number $n$ such that, for any set $F$ of $n$ trees of size $d$, there is an $F$-decomposition of $Q_{d}$, and shows that $n \geq 2$. In the second part of this paper we shall prove that $n \geq 6$.

\section{Balanced decompositions of cubes}

A decomposition of a graph $G$ into a graph $H$ is a system of mutually edge disjoint subgraphs $G_{1}, \ldots, G_{n}$ of $G$ such that $E\left(G_{1}\right) \cup \cdots \cup E\left(G_{n}\right)=E(G)$ and $G_{i}$ is isomorphic to $H$ for $i=1, \ldots, n$. In this paper we deal with decompositions of the $n$-dimensional cube which we denote by $Q_{n}$. There are many ways to represent an $n$ cube. The following one is the most suitable for our purposes. The vertex set is the set $A^{n}$, the set of all ordered $n$-tuples of 0 's and 1 's, and two vertices are connected if they differ in precisely one coordinate. By $O$ and $I$ we denote the $n$-tuples $(0,0, \ldots, 0)$ and $(1,1, \ldots, 1)$ respectively. For $\alpha \in A^{n}$ we denote by $\alpha_{i}$ the $i$ th coordinate of $\alpha$, and $\ell_{i}$ is the $n$-tuple with $\left(\ell_{i}\right)_{i}=1$ and $\left(\ell_{i}\right)_{j}=0$ for $j \neq i$. If $\alpha$ and $\beta$ belong to $A^{n}$, the sum $\alpha+\beta$ is also in $A^{n}$ and is the componentwise sum (mod 2). Further $\alpha \in A^{n}$ is called even or odd according to whether the number of non-zero coordinates of $\alpha$ is even or odd. Finally, let $G$ be a graph of size $n$. Then a decomposition 
$D=\left\{G_{1}, \ldots, G_{2^{n-1}}\right\}$ of $Q_{n}$ into $G$ is said to be balanced if there exist isomorphisms $\varphi_{i}: G \rightarrow G_{i}$ such that, for any $v \in V(G),\left\{\varphi_{i}(v), i=1, \ldots, 2^{n-1}\right\}$ coincides either with the set of all even vertices of $Q_{n}$ or with the set of all odd vertices of $Q_{n}$.

Clearly, if there is a balanced decomposition of $Q_{n}$ into $G$ then there is a balanced decomposition of $Q_{n}$ into $G$ such that for a given vertex $w$ of $G$ the images of $w$ under the $\varphi_{i}$ 's occupy all even vertices of $Q_{n}$.

LEMMA 1 . There is a balanced decomposition of $Q_{2 n}$ into cycles of length $2 n$.

ProOF. Suppose first that $n$ is even, say $2 n=4 k$. Let $T$ and $T^{\prime}$ denote the vertices $T=\ell_{1}+\ell_{2}+\cdots+\ell_{2 k}$ and $T^{\prime}=\ell_{2 k+1}+\cdots+\ell_{4 k}$. Consider two $4 k$-cycles of $C_{1}$ and $C_{2} Q_{4 k}$, where

$C_{1}=O, \ell_{1}, \ell_{1}+\ell_{2}, \ldots, T, T+\ell_{1}, T+\ell_{1}+\ell_{2}, \ldots, T+\ell_{1}+\ell_{2}+\cdots+\ell_{2 k}(=O)$

and $C_{2}$ is obtained from $C_{1}$ by exchanging any $\ell_{i}$ in the definition of $C_{1}$ with $\ell_{i+2 k}$ (that is, $C_{2}$ is obtained from $C_{1}$ by cyclicly shifting the coordinates of any vertex of $C_{1}$ by $2 k$ to the right). For example, if $d=8$, where $d=2 n$ is the dimension of the cube,

$\begin{array}{ccc}C_{1} \quad 00000000 & C_{2} & 00000000 \\ 10000000 & & 00001000 \\ 11000000 & & 00001100 \\ 11100000 & & 00001110 \\ 11110000 & & 00001111 \\ 01110000 & & 00000111 \\ 00110000 & & 00000011 \\ 00010000 & & 00000001 \\ 00000000 & & 00000000\end{array}$

It is obvious that the mapping $\varphi_{\alpha}: A^{d} \rightarrow A^{d}$, defined by $\varphi_{\alpha}(\beta)=\beta+\alpha$ for $\beta \in A^{d}$, is an automorphism of $Q_{d}$. This implies that $C_{i}+\alpha$ is a $d$-cycle of $Q_{d}$ for any $\alpha \in A^{d}, i=1,2$. To finish the proof we show that $\mathscr{C}=\left\{C_{1}+\alpha ; \alpha \in A^{d}, \alpha\right.$ is even, $\left.\alpha_{2 k}=0\right\} \cup\left\{C_{2}+\beta ; \beta \in A^{d}, \beta\right.$ is even, $\left.\beta_{4 k}=0\right\}$ is a decomposition of $Q_{d}$ in $C_{d}$ 's.

As $\mathscr{C}$ contains $2^{d-1}$ cycles it suffices to prove that they are edge disjoint. Suppose, to the contrary, that there is an edge $f$ of $Q_{d}$ which belongs to two different cycles of $\mathscr{C}$.

We consider two cases. 
CASE 1. There exist $\alpha, \beta \in A^{d}, \alpha \neq \beta$, such that $f$ belongs to both $C_{1}+\alpha$ and $C_{1}+\beta$. Let $f=s t$, where $s=t+\ell_{j}$, that is, $s$ and $t$ differ precisely in the $j$ th coordinate. In $C_{1}$, there are two edges such that their end vertices differ in the $j$ th coordinate; denote them by $g_{1}=v_{1} w_{1}, g_{2}=v_{2} w_{2}$. Then $f$ must be the image of $g_{1}$ or $g_{2}$. By the definition of $C_{1}$ we can assume

$$
\begin{aligned}
& v_{1}+v_{2}=w_{1}+w_{2}=T, \\
& v_{1}+w_{2}=v_{2}+w_{1}=\ell_{j}+T .
\end{aligned}
$$

Hence, either

$$
f=g_{1}+\alpha=g_{1}+\beta
$$

or

$$
f=g_{1}+\alpha=g_{2}+\beta .
$$

In the former case: either $s=v_{1}+\alpha=v_{1}+\beta$, implying $\alpha=\beta$, which is impossible; or $s=v_{1}+\alpha=w_{1}+\beta$ (whence $v_{1}+w_{1}=\alpha+\beta$ ), so $\ell_{j}=\alpha+\beta$, contradicting the fact that $\alpha+\beta$ is even. In the latter case: either $s=v_{1}+\alpha=v_{2}+\beta$, and by (1), $\alpha+\beta=T$, contradicting $\alpha_{k}=\beta_{k}=0$; or $s=v_{1}+\alpha=w_{2}+\beta$, which, by (2), yields $T+\ell_{j}=\alpha+\beta$ contradicting $\alpha+\beta$ is even. So case 1 is impossible.

CASE 2. There exist $\alpha, \beta \in A^{d}$ such that $f=s t$ belongs to $C_{1}+\alpha$, implying $s$ and $t$ differ in the $j$ th coordinate, $j \leq 2 k$, and also $f$ belongs to $C_{2}+\beta$ implying $s$ and $t$ differ in the $j$ th coordinate for $j>2 k$. So case 2 is impossible.

To finish the proof it is necessary to show that $\mathscr{C}$ is a balanced decomposition. Because of the symmetry of a cycle and the way we have defined $C$ it is sufficient to pick, for any cycle $C \in \mathscr{C}$, a vertex $v_{C} \in C$ such that the set $\left\{v_{C} ; C \in \mathscr{C}\right\}$ is the set of all even vertices of $Q_{d}$. It is a matter of routine to verify that the following choice has that property:

$$
\begin{aligned}
& \text { For } C_{1}+\alpha \text { pick } O+\alpha \text { if } \alpha_{4 k}=0 ; \text { otherwise pick } T+\alpha . \\
& \text { For } C_{2}+\alpha \text { pick } O+\alpha \text { if } \alpha_{2 k}=1 ; \text { otherwise pick } T^{\prime}+\alpha .
\end{aligned}
$$

Suppose now $2 n=4 k+2$. We represent $Q_{d}$ as in Figure 1 , where the four squares stand for copies of $Q_{d-2}$ induced by $d$-tuples with the same last two coordinates. These last two coordinates are written down above each cube. Consider the decomposition of $Q_{d-2}$ into cycles of length $d-2$ given in the first part of the proof. We take the same decomposition for all four $Q_{d-2}$ 's. By means of these decompositions we generate a decomposition of $Q_{d}$ into cycles of length $d$. We write $v_{i j}$ for the vertex $v$ from the $Q_{d-2}$ whose last two coordinates are $i j$. 


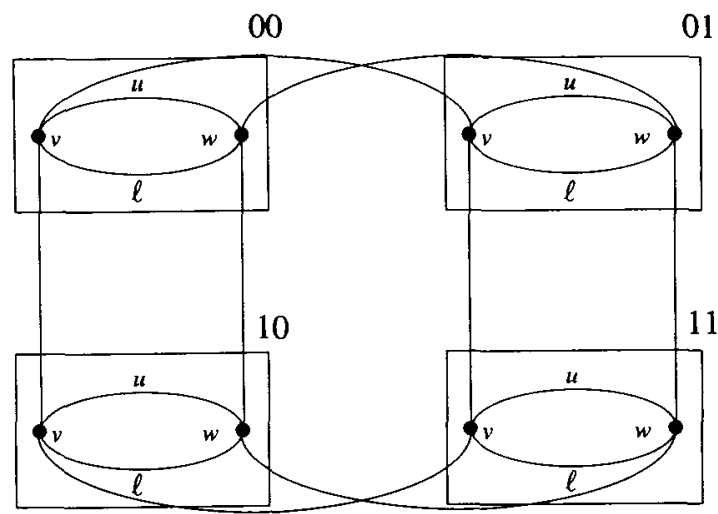

FIGURE 1.

Consider a cycle of $\mathscr{C}$ which is of the form $C_{1}+\alpha$. One of these is depicted in Figure 1. By $v u w(v \ell w)$ we denote the 'upper' ('lower') part of the cycle. Then the cycle generates four cycles of length $d$, namely

$$
\begin{array}{ll}
K_{1}=v_{00} u w_{00} w_{01} u v_{01} v_{00} ; & K_{2}=v_{00} \ell w_{00} w_{10} \ell v_{10} v_{00} \\
K_{3}=v_{10} u w_{10} w_{11} u v_{11} v_{10} ; & K_{4}=v_{01} \ell w_{01} w_{11} \ell v_{11} v_{01}
\end{array}
$$

We choose $v$ and $w$ be the vertices $O+\alpha$ and $T+\alpha$, respectively. Clearly, the set of vertices $\left\{O+\alpha, T+\alpha ; \alpha\right.$ is even, $\left.\alpha_{2 k}=0\right\}$ is the set of all even vertices of $Q_{d-2}$. On the other hand, consider a cycle of $\mathscr{C}$ which is of the form $C_{2}+\alpha$. In this case we choose as $v$ and $w$ the vertices $O+\alpha+\ell_{1}$ and $T^{\prime}+\alpha+\ell_{1}$, respectively. Then the set $\left\{O+\alpha+\ell_{1}, T^{\prime}+\alpha+\ell_{1} ; \alpha\right.$ is even, $\left.\alpha_{4 k}=0\right\}$ is the set of all odd vertices of $Q_{d-2}$. Hence, the cycles generated in the above stated manner form a decomposition $D$ of $Q_{d}$.

We now show that the decomposition is balanced. From the way we constructed the decomposition, it is sufficient to pick from any $C \in D$ a vertex $v_{C}$ and that $\left\{v_{C} ; C \in D\right\}$ is the set of all even vertices of $Q_{d}$. In the case that the underlying cycle from $\mathscr{C}$ is of the form $C_{1}+\alpha$ we choose as $v_{C}$ the vertex $v_{00}$ for $K_{1}, w_{00}$ for $K_{2}, v_{11}$ for $K_{3}$, and $w_{11}$ for $K_{4}$. For a cycle of $\mathscr{C}$ of the form $C_{2}+\alpha$ we choose $v_{01}$ for $K_{1}$, $w_{10}$ for $K_{2}, v_{01}$ for $K_{3}$, and $w_{01}$ for $K_{4}$.

\section{Decomposing a cube into a graph}

Our first main result is the following generalization of the theorem of Fink, Jacobson, Truzczyński and Tuza. 
THEOREM 2. Let $G$ be a graph of size $n$, each block of which is either a cycle or an edge. If $G$ is embeddable into $Q_{n}$ then $Q_{n}$ can be decomposed into $G$.

REMARK. Since $Q_{n}$ is bipartite $G$ is embeddable into $Q_{n}$ if and only if each cycle of $G$ has even length.

PROOF. We proceed by induction on the number of blocks of $G$. To be able to carry out the second step of the induction we prove a stronger statement, namely that there is a balanced decomposition of $Q_{n}$ into $G$.

Firstly, suppose the number $m$ of blocks equals 1 . If $G$ is a single edge the statement is obvious. If $G$ is a cycle then it must be of even length and the claim follows from Lemma 1.

Now we assume $m>1$. Suppose first that $G$ is connected and let $w$ be a cutpoint of $G$. We split $G$ at $w$ into two connected subgraphs $F$ and $H$ of sizes $k$ and $n-k$ respectively. Any block of $G$ belongs entirely to $F$ or entirely to $H$ and $w$ is the only vertex which belongs to both $F$ and $H$. Now we consider two decompositions $A$ and $B$ of the set of vertices of $Q_{n} . A$ is a decomposition into $2^{k}$ classes where two vertices of $Q_{n}$ belong to the same class when their last $k$ coordinates coincide. $B$ has $2^{n-k}$ classes; two vertices are in the same class of $B$ when their first $n-k$ coordinates coincide. Clearly, the subgraph of $Q_{n}$ induced by any class in $A$ is an $(n-k)$-dimensional cube. Analogously, a $k$-dimensional cube is induced by any class in $B$.

It is straightforward that the $2^{k}+2^{n-k}$ cubes induced by $A$ and $B$ form an edgedecomposition of $Q_{n}$. Take a balanced decomposition into $H$ of any $(n-k)$ dimensional cube $Q^{*}$ given by a class of $A$ and a balanced decomposition into $F$ of any $k$-dimensional cube $Q^{*}$ given by a class of $B$ such that the images of the vertex $w$ occupy even vertices. (Note that $Q^{*}$ is a $k$ - (or $(n-k)$-) dimensional cube but any of its vertices has $n$ coordinates and the phrase 'even vertex' refers to the number of non-zero coordinates in this description of vertices of $Q^{*}$.) Thus we get $2^{n-1}$ subgraphs of $G$ isomorphic to $F$ and $2^{n-1}$ subgraphs of $G$ isomorphic to $H$ with vertex $w$ occupying any even vertex twice.

For each even vertex, take the subgraphs isomorphic to $F$ and $H$ which have that vertex as $w$ and paste them together (at $w$ ) to form a graph isomorphic to $G$. Then these graphs form a decomposition of $Q_{n}$ into $G$, and clearly it is balanced.

If $G$ is disconnected we proceed as above, where $F$ is a component of $G$ and $H=G-F$, and we skip over the last step of pasting copies of $F$ and $H$.

It is obvious from the proof that Theorem 2 could be strengthened in the following way: let $\mathscr{H}$ be a family of graphs such that for any graph $H \in \mathscr{H}$ there is a balanced decomposition of $Q_{|H|}$ into $H$. Then for any graph $G$ all of whose blocks are from $\mathscr{H}$ there is a balanced decomposition of $Q_{|G|}$ into $G$. 


\section{Decomposing a cube into a family}

In order to prove our theorem on decomposing cubes into families of trees, we need a result on decomposing the union of two disjoint copies of $Q_{3}$ into rooted trees of size 3. We consider the set $S=\left\{P_{3}^{m}, P_{3}^{t}, C^{c}, C^{p}\right\}$, where:

$P_{3}^{m}$ is a path of length 3 rooted at a midpoint;

$P_{3}^{t}$ is a path of length 3 rooted at a terminal point;

$C^{C}$ is a claw of size 3 rooted at the center;

$C^{p}$ is a claw of size 3 rooted at a pendant vertex.

We write $Q_{3}^{1}$ and $Q_{3}^{2}$ for two disjoint copies of $Q_{3}$, with vertices $\left\{000^{1}, \ldots, 111^{1}\right\}$ and $\left\{000^{2}, \ldots, 111^{2}\right\}$ respectively. $H=Q_{3}^{1} \cup Q_{3}^{2}$.

LEMMA 3. Suppose $\mathscr{F}^{1}$ is the collection $\left\{T_{1}, T_{2}, T_{3}, T_{4}, T_{5}, T_{6}\right\}$, where each $T_{i}$ is a member of $S$. Then one can choose $T_{7}$ and $T_{8}$ in $\mathscr{F}^{1}$ so that there is a decomposition $H=\bigcup_{i=1}^{8} T_{i}$ with the property that the roots of the $T_{i}$ which lie in $Q_{3}^{1}$ form a set $V^{1}$ and the roots of the $T_{i}$ which lie in $Q_{3}^{2}$ form a set $V^{2}$ where $V^{2}=\left\{v^{2}: v^{1} \notin V^{1}\right\}$.

PROOF. First we state four propositions which can be easily verified by the reader. Denote by $E$ and $O$ the sets of even or odd vertices of $Q_{3}$, respectively.

PROPOSITION 1. For any tree $F$ in $\mathscr{S}$ there is a decomposition of $Q_{3}$ into $F$ so that the roots of the F's occupy the set $E$.

Proposition 2. Let $F_{1}, F_{2}$ be a pair of trees of $\mathscr{S}$ such that $\left\{F_{1}, F_{2}\right\} \neq\left\{C^{c}, C^{p}\right\}$. Then there is a decomposition of $Q_{3}$ into two copies of $F_{1}$ and two copies of $F_{2}$ so that the roots occupy the set $E$.

PROPOSITION 3. Let $\left\{F_{1}, F_{2}\right\}=\left\{C^{c}, C^{p}\right\}$. Then there is a decomposition of $Q_{3}$ into three copies of $F_{1}$ and a copy of $F_{2}$ so that the roots occupy the vertices of the set $S=\{000,100,001,111\}$.

Proposition 4. There is an $\mathscr{S}=\left\{P_{3}^{m}, P_{3}^{t}, C^{c}, C^{p}\right\}$ decomposition of $Q_{3}$ so that the roots occupy the set $E$.

In each case, the symmetry of $Q_{3}$ means that the proposition remains true if the set of root positions is replaced by its complement ( $S$ by its complement in Proposition $3, E$ by $O$ in the others).

To exhibit the required decompositions of $H$ we use the notation

$$
(a, b, c, d) \rightarrow\left(a^{\prime}, b^{\prime}, c^{\prime}, d^{\prime}\right):\left(a_{1}, b_{1}, c_{1}, d_{1}\right)+\left(a_{2}, b_{2}, c_{2}, d_{2}\right) .
$$


Here $(a, b, c, d)$ means that $\mathscr{F}^{\prime}$ contains $a$ copies of $A, b$ copies of $B, c$ copies of $C$ and $d$ of $D$ (where $A, B, C, D$ are $P_{3}^{m}, P_{3}^{t}, C^{c}, C^{p}$ in some order); $a+b+c+d=6$. $\left(a^{\prime}, b^{\prime}, c^{\prime}, d^{\prime}\right)$ exhibits the same information when trees $T_{7}$ and $T_{8}$ are included, so $a^{\prime}+b^{\prime}+c^{\prime}+d^{\prime}=8$. The quadruple $\left(a_{1}, b_{1}, c_{1}, d_{1}\right)$ gives the same information for the trees in $Q_{3}^{1}$, and $\left(a_{2}, b_{2}, c_{2}, d_{2}\right)$ for $Q_{3}^{2}$, so $a_{1}+a_{2}=a^{\prime}$, and so on. For example, $(4,2,0,0) \rightarrow(4,4,0,0):(4,0,0,0)+(0,4,0,0)$ means that in the family $\mathscr{F}^{1}=\left\{T_{i}^{1} ; i=1, \ldots, 6\right\}$ one tree from $\mathscr{S}$ occurs 4 times, one other tree occurs 2 times and the other two are not represented. As $T_{7}^{1}$ and $T_{8}^{1}$ we choose two copies of the tree of $\mathscr{S}$ which is $\mathscr{F}^{1}$ twice. In the decomposition of $H$ we have 4 copies of the first tree in $Q_{3}^{1}$ and four copies of the second tree in $Q_{3}^{2}$. By Proposition 1 we can carry out the first decomposition so that the four roots are the members of $E$, and the second so that the roots are the members of $O$, so the roots have the required property.

Below we list all possibilities and corresponding choices of $T_{7}^{1}$ and $T_{8}^{1}$ and decompositions of $H$.

$$
\begin{aligned}
& (6,0,0,0) \rightarrow(8,0,0,0):(4,0,0,0)+(4,0,0,0) \\
& (5,1,0,0) \rightarrow(6,2,0,0):(4,0,0,0)+(2,2,0,0) \text { otherwise } \\
& :(3,1,0.0)+(3,1,0,0) \quad \text { if }\{A, B\}=\left\{C^{c}, C^{p}\right\} \\
& (4,2,0,0) \rightarrow(4,4,0,0):(4,0,0,0)+(0,4,0,0) \\
& (4,1,1,0) \rightarrow(4,2,2,0):(4,0,0,0)+(0,2,2,0) \quad \text { otherwise } \\
& :(2,2,0,0)+(2,0,2,0) \quad \text { if }\{B, C\}=\left\{C^{c}, C^{p}\right\} \\
& (3,3,0,0) \rightarrow(4,4,0,0):(4,0,0,0)+(0,4,0,0) \\
& (3,2,1,0) \rightarrow(4,2,2,0): \text { as above } \\
& (3,1,1,1) \rightarrow(5,1,1,1):(4,0,0,0)+(1,1,1,1) \\
& (2,2,1,1) \rightarrow(2,2,2,2):(1,1,1,1)+(1,1,1,1) .
\end{aligned}
$$

In each case it follows from Propositions 1-4 that the roots can be placed appropriately.

THEOREM 4. Suppose $\mathscr{D}=\left\{T_{1}, T_{2}, T_{3}, T_{4}, T_{5}, T_{6}\right\}$, where the $T_{i}$ are trees of size $d$. Then $Q_{d}$ decomposes into $\mathscr{D}$.

PROOF. We represent the cube $Q_{d}$ as shown in Figure 2. The eight squares stand for subcubes $Q_{d-3} ; Q_{i j k}$ is formed from all vertices with last three binary digits $i j k$. Any eight vertices with the same first $d-3$ coordinates induce a subcube of dimension 3. One of these $2^{d-3}$ subcubes is represented in the Figure $2 ; v$ is a vertex of $Q_{d-3}$ (the same vertex in each case) and the lines represent the $Q_{3}$. We write $Q_{3}(v)$ for this 3-cube. 


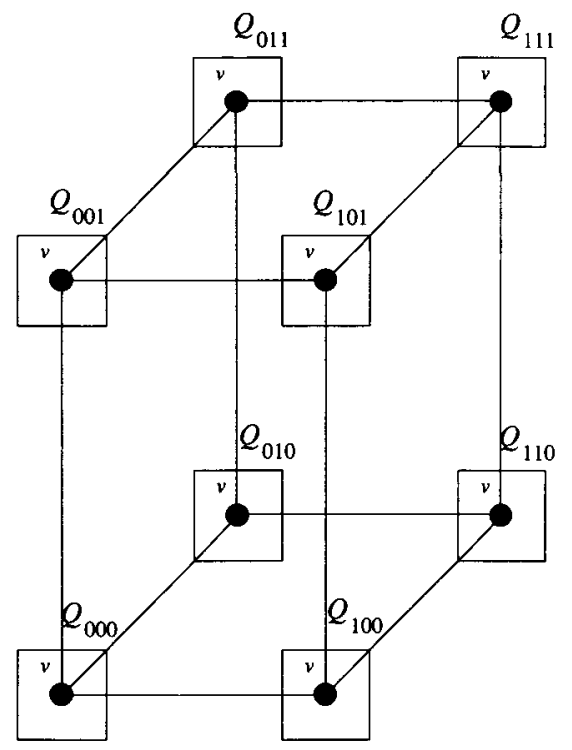

FIGURE 2.

For each $i$ select a vertex $x_{i}$ of $T_{i}$ such that we can split $T_{i}$ at $x_{i}$ into two subtrees $T_{i}^{1}$, of size 3 , and $T_{i}^{2}$. We view $T_{i}^{1}$ and $T_{i}^{2}$ as rooted at $x_{i} ; T_{i}^{1}$ must be isomorphic to one of $P_{3}^{m}, P_{3}^{t}, C^{c}$ or $C^{p}$. Write $\mathscr{F}^{1}=\left\{T_{1}^{1}, T_{2}^{1}, T_{3}^{1}, T_{4}^{1}, T_{5}^{1}, T_{6}^{1}\right\}$. Select two trees $T_{7}^{1}$ and $T_{8}^{1}$ from $\mathscr{F}^{1}$, and find a decomposition of a graph $H=Q_{3}^{1}+Q_{3}^{2}$, as in Lemma 3.

The subcube $Q_{3}(v)$ is decomposed as $Q_{3}^{1}$ if $v$ is an even vertex, and as $Q_{3}^{2}$ if $v$ is odd. For each $i j k$ we choose a balanced decomposition of $Q_{i j k}$ into $T_{s}^{2}$, where $s$ is the index such that the tree whose root was placed at either $i j k^{1}$ or $i j k^{2}$ in the decomposition of $H$ was a $T_{s}^{1}$. (If $s=7$ or 8 we take $T_{s}^{2}$ the tree $T_{r}^{2}$ where $T_{s}^{1}$ is isomorphic to $T_{r}^{1}$.) If it was at $i j k^{1}$ then the roots of the copies of $T_{s}^{2}$ will occupy all the even vertices of $Q_{i j k}$, otherwise they occupy all the odd vertices of $Q_{i j k}$. In either case, at each root we glue together a copy of $T_{s}^{1}$ and $T_{s}^{2}$ to form a member of $\mathscr{D}$. These trees form the desired decomposition of $Q_{d}$.

\section{References}

[1] A. Dolinski and M. Tarsi, 'Graph decomposition is NPC - a complete proof of Holyer's conjecture.', in: Proc. 24th annual ACM symposium on theory of computing (Victoria, BC, 1992) pp. 252-263.

[2] J. F. Fink, 'On the decomposition of n-cubes into isomorphic trees', J. Graph Theory 14 (1990), $405-411$. 
[3] M. S. Jacobson, M. Truszczynski and Z. Tuza, 'Decompositions of regular bipartite graphs', Discrete Math. 89 (1991), 17-27.

[4] G. Ringel, 'Problem 25', in: Theory of graphs and its applications (Smolenice, 1963) (Czechoslovak Academy of Science, Prague, 1964) p. 162.

[5] R. M. Wilson, 'Decompositions of complete graphs into subgraphs', Congr. Numer. 15 (1976), 647-659.

Slovak Technical University

81219 Bratislava

Comenius University

Slovakia

84215 Bratislava

Slovakia

Southern Illinois University

Carbondale

Illinois 62901 